\title{
CYTOLOGICALLY ACTIVE SUBSTANCES PRODUCED BY SOIL MICRO-ORGANISMS
}

\author{
L. L. CAVAlLI-SFORZA, L. PETRELLA and A. GerRA \\ Istituto di Genetica dell'Universita di Milano, Istituto Sieroterapico \\ Milanese Serafino Belfanti
}

Received I5.X.54

Substances showing activities which may be detected by cytological observations are nowadays known from many sources. Broadly speaking, cytological tests permit to discover at least two kinds of action: antimitotic, or altering the cell cycle, and mutagenic, or chromosome breaking. The two actions are not necessarily unrelated, and may be shown by the same substance, but it seems convenient to keep them distinct in a first simple classification.

While some of the substances known are synthetic in origin, and some are extracted from plants (more rarely from animals), a few new antimitotic drugs have been discovered which are produced by Actinomycetes (e.g. actinomycin, Hackmann, 1953). The discovery of mutagens of natural origin (d'Amato, 1950) makes it likely that among metabolic products of micro-organisms not only antimitotics, but also mutagens can be found. In view of these facts, a survey of cytologically active substances from microbial sources was undertaken. Soil micro-organisms, recently isolated, belonging to the classes of Schyzomycetes, Phycomycetes, Ascomycetes and Fungi Imperfecti were employed. Culture filtrates on suitable media were tested for cytological activity by means of the standard test on Allium cepa root tips.

\section{MATERIAL AND METHOD}

Micro-organisms were isolated from samples of soil collected in various places (mostly around Milan) by means of the usual plating procedures on agar media suitable for the isolation of bacteria, actinomycetes, fungi. The production of culture filtrates for use in cytological analysis demanded the choice of a suitable medium, as it was found that most standard media, both synthetic and not, are unfavourable for root growth and greatly decrease the frequency of cell divisions visible on microscopical examination. A medium was selected which, while permitting good growth of most of the strains to be examined, interfered least with the development of root tips, consisting of a carrot infusion broth supplemented with 2 per cent. glucose.

Seven-day-old aerated cultures were filtered, and young roots of onions exposed to the filtrates overnight. Root tips were stained with acetocarmine and squashed after heating to the first boiling.

\section{EXPERIMENTAL RESULTS}

The results of the cytological examination can be briefly related as follows. One type of alteration was fairly commonly observed in the treated roots, namely $\mathrm{C}$-mitotic poisoning. With little variation, at least from a qualitative point of view, a similar picture emerged 
with a variety of filtrates : metaphase blocks reminding more or less closely of those obtained by the action of colchicine. Quantitatively, of course, there was variation from filtrate to filtrate, and a few filtrates showed activities up to complete block of all mitoses observed.

More rarely were other kinds of cytological alterations noticed. Three filtrates showed signs of mutagenic action, chromosome fragments being observed with two filtrates, and chromatin bridges in another instance.

A summary of the activities detected is given in table I, where the I02 filtrates tested are subdivided according to the main systematic groups. As will be seen from the totals, about 22 per cent. of the filtrates showed some degree of cytological activity, most of the cases (I9 per cent. of the total) representing C-mitotic activity.

In almost all groups of micro-organisms tested was some activity found, except in the Phycomycetes, and there perhaps because of the small number examined. The distribution according to systematic

TABLE I

Distribution of micro-organisms showing cytological activity according to main systematic groups. Figures between brackets refer to numbers of strains showing activity other than $C$-mitotic

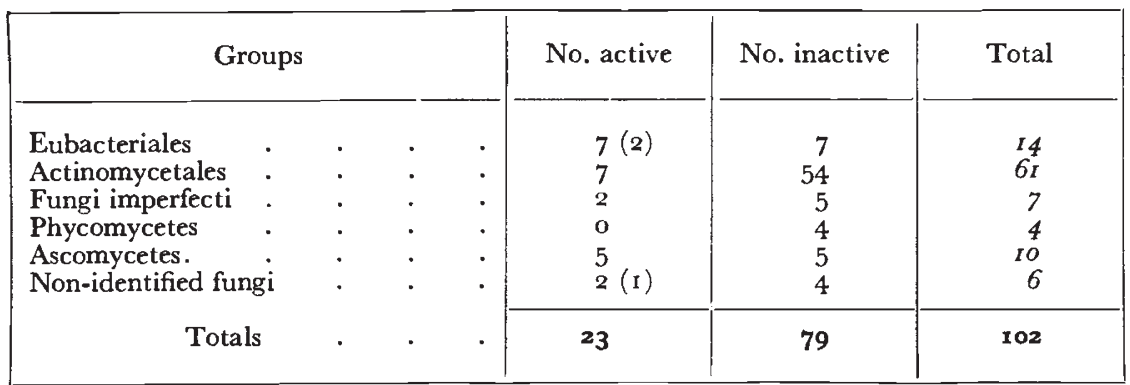

groups shows some heterogeneity $\left(\chi^{2}=16 \cdot 35\right.$ with 5 d.f.), the frequency of active filtrates being significantly smaller in the Actinomycetes. This conclusion is not, however, permissible at this stage, in view of the method of selection of micro-organisms employed. In fact, while for groups other than Actinomycetes, selection was practised in favour of micro-organisms endowed with antibiotic activity, Actinomycetes were sampled at random. The lower frequency of active filtrates in the latter group might therefore be the consequence of a correlation between antibiotic and antimitotic activity, such as would arise if the same substance were often endowed with both properties.

Antibiotic activity was tested by means of eleven indicator strains. A direct analysis of the correlation between it and cytological activity is given in tables 2, 3, of which neither shows significant correlation. On the other hand, these tables are not significantly at variance with the hypothesis that a low correlation exists, of insufficient magnitude to be detected by the use of unselected samples of the 
present size, but capable of affecting the frequency of one type of activity in a significant way, when the other type of activity is selected.

TABLE 2

Correlation between antibiotic and antimitotic activity in Actinomycetes

\begin{tabular}{|c|c|c|c|c|c|}
\hline & & & \multicolumn{2}{|c|}{ Antibiotic activity } & \multirow{2}{*}{ Total } \\
\hline & & & + & - & \\
\hline \multirow[t]{2}{*}{ Antimitotic activity } & \multirow[t]{2}{*}{$\cdot \quad \cdot\}$} & \multirow[t]{2}{*}{ \pm} & $\begin{array}{l}2 \\
5\end{array}$ & $\begin{array}{l}\text { I I } \\
43\end{array}$ & $\begin{array}{l}13 \\
48\end{array}$ \\
\hline & & & 7 & 54 & $6 x$ \\
\hline
\end{tabular}

TABLE 3

Correlation between antibiotic and antimitotic activities in micro-organisms other than Actinomycetes

\begin{tabular}{|c|c|c|c|c|c|}
\hline & & & \multicolumn{2}{|c|}{ Antibiotic activity } & \multirow{2}{*}{ Total } \\
\hline & & & + & - & \\
\hline \multirow[t]{2}{*}{ Antimitotic activity } & \multirow[t]{2}{*}{$\cdot \quad \cdot\}$} & \multirow[t]{2}{*}{ \pm} & $\begin{array}{r}9 \\
19\end{array}$ & $\begin{array}{l}4 \\
6\end{array}$ & $\begin{array}{l}13 \\
25\end{array}$ \\
\hline & & & 28 & IO & $3^{8}$ \\
\hline
\end{tabular}

TABLE 4

Correlation between antibiotic activity against single indicator strains and antimitotic activity

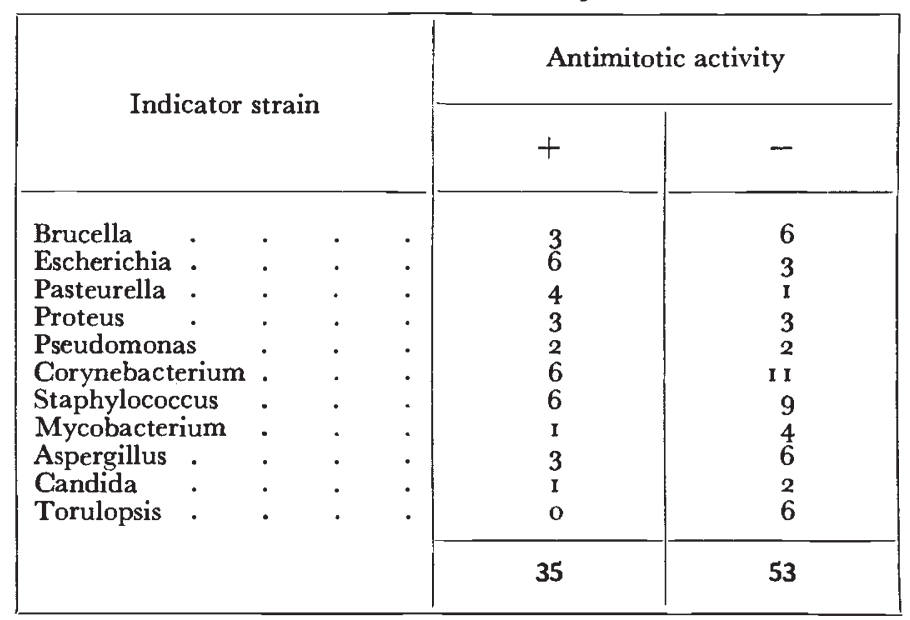

The problem of the correlation between antibiotic and antimitotic activity was tested further by analysis for the single indicator strains, as given in table 4. There is no significant heterogeneity $\left(\chi^{2}=7 \cdot 64\right.$ 
with Io d.f.) ; but, on grouping the indicator strains according to the simplest scheme, i.e. into Gram positives, Gram negatives, and others, some heterogeneity appears (table $5 ; \chi^{2}=6 \cdot 20$ with 2 d.f., $\mathrm{P}<5$ per cent.) pointing to a slight correlation between action against Gram negative micro-organisms, and in the Allium test. It is clear, however, that this point would need further investigation.

The active strains were classified into the following genera :

Eubacteriales : Bacillus, Corynebacterium, Micrococcus.

Actinomycetales: Actinomyces and Streptomyces.

Moniliales : Fusarium, Stysanus.

Ascomycetes : Aspergillus (glaucus, fumigatus) ; Penicillium (spinulosum, and an unidentified Penicillium of the asymmetrical group).

Further tests were made to inquire whether the particular medium employed was responsible for the high frequency of antimitotics

TABLE 5

Correlation between antimitotic activity and antibiotic activity against certain groups of micro-organisms

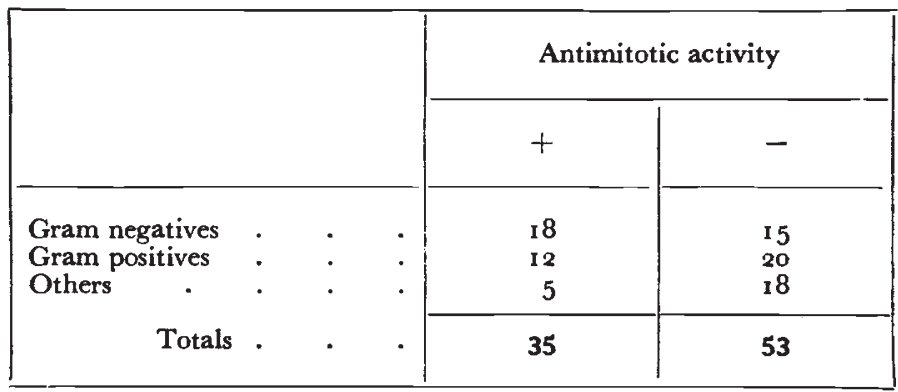

produced. Seven active strains were re-tested after growth on a synthetic medium plus yeast extract and none failed to reproduce the activity in the new conditions.

Finally, it was tested whether the C-mitotic action observed could lead to doubling of the chromosome complement of the cell. Onions, the roots of which had been exposed overnight to the action of filtrate 474 were placed again into tap water and examined after various times. Several polyploid mitoses were observed. It can therefore be asserted that some at least of the active principles can act as polyploidising agents.

\section{DISCUSSION}

The production of substances active on the cell cycle of higher organisms in cultures of micro-organisms isolated from the soil seems interesting under several points of view. Firstly, it is perhaps surprising to find that metabolic products of micro-organisms are so often endowed with selective toxicity for the reproduction processes of the 
cells of higher organisms. It is of interest to compare their frequency (about 20 per cent.) with that with which antibiotic activity is detected (from unpublished data on $\mathbf{8 4 7}$ micro-organisms of origin and distribution comparable with that of the strains used in the present survey) :

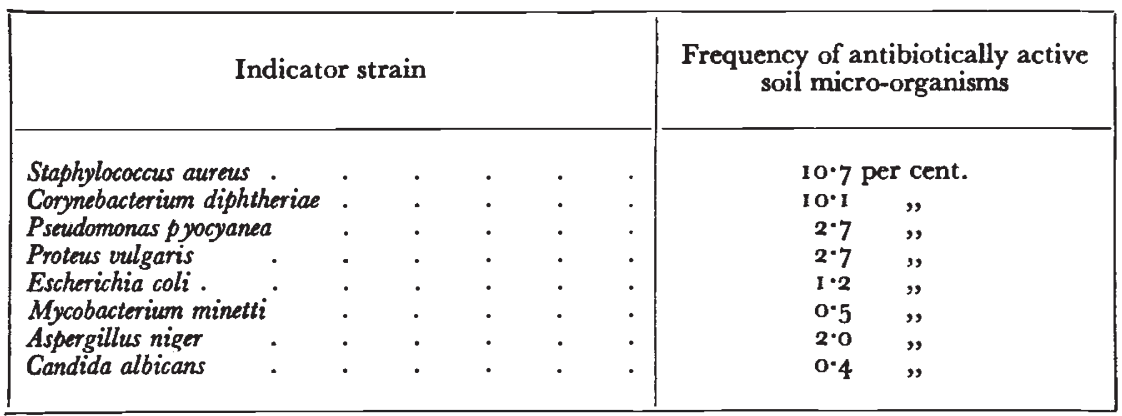

These frequencies are so largely dependent upon the method used for the test that most conclusions based on them would seem unwarranted, but it seems at least permissible to conclude that antimitotic compounds are produced by micro-organisms at a rate similar, or higher than that of antibiotics.

A second consideration of general interest arising from the present research is that micro-organisms normally present in the soil are likely to be active determiners of polyploidisation in plants. It is obviously difficult to distinguish between "spontaneous" polyploidisation and that induced by agents of microbial origin, except by special experiments. But, at the present stage, one would see no reason why this particular kind of interaction should not take place in nature as well. The main limiting factor is, of course, the concentration of active substances that can be reached in the soil. The high numbers of germs found in the soil, and the frequency with which active producers of antimitotics are available, make it likely that sufficient concentrations are often attained.

The relative infrequency of mutagenic agents may also deserve some comment. Permeability conditions are, of course, to be kept in mind as a possible source of bias. Change of cultural conditions might also seriously affect the picture. Finally, the presence of natural antimutagens might obscure it.

A further point to be stressed is the desirability of using cytological tests in screening programmes, in the search of drugs active against diseases which, like cancer and leukemias, are the result for abnormal cell proliferation. There is already proof that drugs of this kind can be produced by micro-organisms. The frequency with which antimitotics can be found encourages us to believe that a wealth of new substances, some of which may be useful in human therapy, is thus made available. Cytological tests may prove very useful in this connection; even if they are more laborious than screening methods for antibiotics, they are certainly far easier than methods using 
experimental tumours. Should an antimitotic turn up to be antibiotic, the task of chemical isolation would certainly be simplified, but the chances for this seem to be fairly low.

\section{CONCLUSIONS AND SUMMARY}

Metabolic products of micro-organisms are found showing antimitotic activity with a rather high frequency. Active strains were obtained belonging to the Eubacteriales, Actinomycetales, Moniliales, and Ascomycetes, by means of a screening test using Allium cepa root tips. Mutagenic action was less frequent and striking. The correlation of antibiotic and antimitotic activity is not clear-cut and, if any, cannot be high. Antimitotic agents from soil micro-organisms are likely to act as powerful polyploidising agents in nature, probably explaining at least part of the "spontaneous" origin of polyploids. The advantages of cytological screening tests in the search for antimitotic drugs have been briefly discussed.

Acknowledgments.-We wish to express our gratitude to Prof. C. Barigozzi for his constant supervision of the cytological work and for his interest in the present investigation.

\section{REFERENCES}

HACKMANN, CH. 1953. HBF 386 (Actinomycin C), ein neues cytostatisch wirksamer Naturstoff. Strahlenther, 9o, 296.

D'Amato, F. 1950. The quantitative study of mitotic poisons by the Allium cepa Test : data and problems. Protoplasma, 39, 423.

D'Amato, F. 1950. Recenti acquisizioni nel campo della chemiogenetica: i prodotti del ricambio organico come agenti mutageni. Caryologia, 3, 249 . 